\title{
AVALIAÇÃO ECONÔMICA DA REGENERAÇÃO DA VEGETAÇÃO DE CERRADO, SOB DIFERENTES REGIMES DE MANEJO ${ }^{1}$
}

Antônio Donizette de Oliveira², Anabel Aparecida de Mellloº $^{3}$ José Roberto Soares Scolforo², José Luiz de Pereira Resende $^{2}$ e Josefina Ivonete Fagundes Melo ${ }^{4}$

\begin{abstract}
RESUMO - Os objetivos deste estudo foram avaliar a viabilidade econômica de manejar a vegetação nativa do cerrado submetida a seis diferentes níveis de intervenção, levando-se em conta alterações nos parâmetros valor da terra, nível de produtividade, custo de produção e preço da madeira, e comparar, em termos econômicos, duas opções para uso de terras originalmente ocupadas com vegetação de cerrado: produção de madeira (lenha) para energia, manejando a vegetação do cerrado, e retirada da vegetação para plantio de eucalipto. Os dados foram obtidos em Coração de Jesus-MG, em experimento instalado em uma área de 30 ha, submetida a seis tratamentos (retirada de 50, 70, 80, 90 e 100\% da área basal e testemunha), com cinco repetições cada. Para avaliação econômica usou-se o valor presente líquido, considerando um horizonte de planejamento infinito. Concluiu-se que o ciclo de corte ótimo econômico é de 10 anos. Todos os regimes de manejo foram viáveis economicamente, exceto a retirada de $50 \%$ da área basal. O custo da terra é significativo na formação do custo de produção da vegetação do cerrado, o que evidencia que planos de manejo podem ser mais lucrativos se forem implantados em regiões onde o preço da terra é baixo. Variações na produtividade, nos custos de produção e no preço da madeira também afetaram de maneira significativa a viabilidade econômica dos regimes de manejo. Do ponto de vista econômico, investir no plantio de eucalipto em regiões de cerrado, visando produzir madeira para energia, só é mais interessante que manejar a vegetação do cerrado se a produtividade do eucalipto for maior do que 45 st/ha.ano.
\end{abstract}

Palavras-chave: Análise econômica, floresta nativa, cerrado e ciclo de corte.

\section{ECONOMIC EVALUATION OF SAVANNAH REGENERATION UNDER DIFFERENT MANAGEMENT REGIMES}

\begin{abstract}
The objectives os this study were to evaluate the economic viability of managing a native savannah vegetation, submitted to six different intervention levels, considering changes in: land price, productivity level, production cost and wood price and to compare two options of using land originally covered with savannah vegetation: wood production (firewood) for energy, by managing the savannah vegetation and clearing the vegetation for eucalyptus plantation. The data were obtained in the Alvação Farm, district of Coração de JesusMG. The experiment was installed in an area of 30 hectares, and submitted to 6 treatments (remotion of 50\%, $70 \%, 80 \%, 90 \%$ and $100 \%$ of the basal area, plus control), with 5 repetitions each. For economic evaluation, Net Present Value was used, considering an infinite planning horizon. It was concluded that the optimum economic cycle was 10 years. All the other management regimes were economically viable, except that in which $50 \%$ of the basal area was removed. Land cost is significant in the formation of the production cost of the savannah vegetation, suggesting that managing this vegetation can be more profitable in areas where land price is low; variations in productivity, production costs and wood price also affected significantly the economic viability of all management regimes. Investing in eucalyptus plantation in savannah areas to produce wood for energy is better than managing savannah vegetation only if productivity is over 45 st/ha.year.
\end{abstract}

Key words: $\quad$ Economic analysis, savannah, native forest and cutting cycle.

1 Recebido para publicação em 28.10.1999.

Aceito para publicação em 03.12.2002.

2 Prof. do Dep. de Ciências Florestais da Universidade Federal de Lavras - UFLA, 37200-000 Lavras-MG, <donizete@ufla.br>, <scolforo@ufla.br>, <jlpresen@ufla.br>. ${ }^{3}$ Engenheira Florestal, M.S., aluna do Curso de Doutorado em Engenharia Florestal da Universidade Federal do Paraná - UFPr, Curitiba-PR. ${ }^{4}$ Engenheira Florestal, Supervisora Regional Norte do IEF, Rua Dona Eva 20, Centro, 39400-009 Montes Claros-MG, <ernsup@ief.mg.gov.br>. 


\section{INTRODUÇÃO}

Desde a criação da política de incentivos fiscais a atividade florestal nas áreas de cerrado tem sido bastante intensa, e seu maior objetivo é atender à demanda de carvão vegetal do País (26 milhões $\mathrm{de}^{3}$ ), principalmente do Estado de Minas Gerais (19,5 milhões de $\mathrm{m}^{3}$ ). As áreas exploradas são formadas de vegetação nativa e, ou, plantio de Eucalyptus (ABRACAVE, 1998).

Segundo Cabral (1990), foi a partir do início da década de 70, com a certeza do esgotamento das reservas de petróleo, que se procurou intensificar o uso de outras fontes alternativas de energia, tais como: o carvão mineral, o gás natural e a madeira. No Brasil, a madeira passou a ser considerada como fonte energética nos mais diversos setores industriais.

A exploração da vegetação nativa para produção de carvão vegetal não é acompanhada pela reposição da vegetação. O Estado de Minas Gerais possui longa tradição no uso de carvão vegetal na siderurgia, e uma grande parte da demanda (30\%) ainda é atendida pela exploração irracional do cerrado (Abracave, 1998).

De acordo com Rezende et al. (1986), Minas Gerais contava com mais de 1,5 milhão de hectares de Eucalyptus spp. plantados, que naquela época apresentavam-se com produtividade aquém do esperado, devido à baixa fertilidade do solo, às técnicas de plantio usadas e às espécies plantadas. Sendo assim, em muitas regiões os plantios de eucalipto não são economicamente viáveis, o que torna mais fácil explorar o cerrado, uma vez que neste não há custos de implantação, de produção de mudas e de fertilização. No entanto, isto não significa que a produção de carvão via cerrado seja mais econômica do que a produção via eucalipto, pois o rendimento em carvão por estere de lenha é mais baixo para o cerrado e os custos de exploração e manuseio são mais altos, devido à heterogeneidade das espécies; à desuniformidade de diâmetros; à forma do fuste; às condições de trabalho na área; à altura média das árvores e a distribuição das árvores na área. Esta situação foi, em parte, revertida. Os plantios de eucalipto apresentam produtividades maiores e custos de implantação menores. Torna-se necessário, então, realizar novos estudos sobre a viabilidade econômica dos plantios de eucalipto e de manejo da vegetação do cerrado.

Há poucos estudos que tratam da economicidade de se produzir madeira (lenha) para carvão usando a vegetação do cerrado e que comparem esta opção com aquela em que a madeira para carvão advém de plantios de eucalipto. Nesta linha de pesquisa podem ser citados os trabalhos de Rezende et al. (1986) e Leite (1998).

Os objetivos principais deste estudo foram avaliar a viabilidade econômica de manejar a vegetação nativa do cerrado, com base em diferentes níveis de intervenção, levando em conta alterações nos parâmetros valor da terra, nível de produtividade, custo de produção e preço da madeira; e comparar, em termos econômicos, duas opções para uso de terras originalmente ocupadas com vegetação de cerrado: produção de madeira (lenha) para energia, manejando a vegetação do cerrado, e retirada da vegetação para o plantio de eucalipto.

\section{MATERIAL E MÉTODOS}

A área de estudo está localizada na fazenda Alvação, de propriedade da Reflorestadora do Alto Jequitinhonha (Refloralje), município de Coração de Jesus, Estado de Minas Gerais. A vegetação local é aquela de cerrado stricto sensu.

\subsection{Base de Dados}

No ano de 1986, o Instituto Estadual de Florestas (IEF) instalou, na fazenda Alvação, um experimento com seis diferentes tratamentos, ou seja, retirada de 50, 70, 80,90 e $100 \%$ da área basal total, além do estabelecimento de um tratamento como testemunha. A área ocupada pelo experimento foi de 30 ha e os tratamentos foram distribuídos aleatoriamente em cinco blocos, totalizando 30 talhões de 1 ha cada um.

Em fevereiro de 1996 foi realizado um segundo inventário, onde foram medidas todas as plantas que possuíam circunferência a $30 \mathrm{~cm}$ de altura $(\mathrm{CAS}) \geq 9,5 \mathrm{~cm}$, em cada parcela. As medidas obtidas foram circunferência a $30 \mathrm{~cm}$ de altura (CAS) e a 1,30 $\mathrm{m}$ de altura (CAP), com a utilização de fita métrica, altura do fuste e altura total com a utilização da vara telescópica. Todas as plantas medidas foram marcadas com placas de alumínio e pintadas com tinta vermelha à altura do CAS, para facilitar futuras medições. Para cada indivíduo amostrado foi coletado material botânico, sendo este numerado e herbarizado. A identificação taxonômica foi feita a partir de consultas a especialistas e de visitas a herbários.

Um terceiro inventário foi realizado em junho de 1998. As árvores marcadas foram novamente medidas, 
assim como as plantas foram recrutadas, ou seja, aquelas que atingiram dimensão mínima para medição. Estas plantas foram marcadas com plaquetas de alumínio e pintadas. Para realização deste estudo, foram consideradas as árvores com CAP $\geq 15,7 \mathrm{~cm}$. Foram também contabilizados a mortalidade e o recrutamento ocorrido nos anos de 1996 e 1998, cujo período foi de 2 anos e 4 meses.

A projeção da estrutura da floresta foi de acordo com Pulz et al. (1999) e Buongiorno \& Michie (1980), como se segue:

$$
Y_{t+\Delta t}=G \cdot Y_{i t}+I_{i t}
$$

em que

$Y_{t+\Delta t}=$ número de árvores projetadas; $G=$ probabilidade de transição por classe diamétrica; $Y_{i t}=$ freqüência da classe de diâmetro; e $I_{i t}=$ recrutamento.

A forma matricial da expressão 1 é:

$$
\left[\begin{array}{c}
Y_{1 t+\Delta t} \\
Y_{2 t+\Delta t} \\
Y_{3 t+\Delta t} \\
\cdot \\
\cdot \\
\cdot \\
Y_{n t+\Delta t}
\end{array}\right]=\left[\begin{array}{ccccccc}
a_{1} & 0 & 0 & 0 & 0 & \cdots & 0 \\
b_{2} & a_{2} & 0 & 0 & 0 & \cdots & 0 \\
c_{3} & b_{3} & a_{3} & 0 & 0 & \cdots & 0 \\
0 & c_{4} & b_{4} & a_{4} & 0 & \cdots & 0 \\
0 & 0 & c_{5} & b_{5} & a_{5} & \cdots & 0 \\
\vdots & \vdots & \vdots & \ddots & \ddots & \ddots & 0 \\
0 & 0 & 0 & 0 & c_{n} & b_{n} & a_{n}
\end{array}\right]
$$

$$
*\left[\begin{array}{c}
Y_{1 t} \\
Y_{2 t} \\
Y_{3 t} \\
\cdot \\
\cdot \\
Y_{n t}
\end{array}\right]+\left[\begin{array}{c}
I_{1 t} \\
I_{2 t} \\
I_{3 t} \\
\cdot \\
\cdot \\
I_{n t}
\end{array}\right]
$$

em que $i_{n}=$ i-ésima classe de diâmetro; $a_{i}, b_{i}, c_{i}=$ são as probabilidades de uma árvore viva permanecer na mesma classe diamétrica $\left(a_{i}\right)$, mudar para a classe diamétrica subseqüente $\left(b_{i}\right)$, ou ainda mudar duas classes $\left(c_{i}\right)$. Estas probabilidades foram obtidas como: $a_{i}=$ número de árvores vivas que permaneceram na i-ésima classe diamétrica no período de tempo $\left(\Delta \mathrm{t}=t_{2}-t_{1}\right) /$ número de árvores existentes na i-ésima classe diamétrica no tempo $t_{1} ; b_{\mathrm{i}}=$ número de árvores vivas que migraram da i-ésima classe diamétrica para a i-ésima classe diamétrica +1 no período de tempo $\left(\Delta t=t_{2}-t_{1}\right) /$ número de árvores existentes na i-ésima classe diamétrica no tempo $t_{1} ; c_{i}=$ número de árvores vivas que migraram da i-ésima classe diamétrica para a i-ésima classe diamétrica +2 no período de tempo $\left(\Delta t=t_{2}-t_{1}\right) /$ número de árvores existentes na i-ésima classe diamétrica no tempo $t_{1}$.

em que $t_{1}=$ início do período de crescimento considerado; $t_{2}=$ fim do período de crescimento considerado; e $\Delta t=$ intervalo de tempo entre o início e o fim do período de crescimento considerado $\left(t_{2}-t_{1}\right)$.

Seja para $a_{i}, b_{i}$ e $c_{i}$, a condição é que a árvore mantenha-se viva e não seja colhida no intervalo de tempo considerado.

Deve-se destacar que se for efetuada a projeção da estrutura da floresta para dois períodos de tempo então a expressão (1) evolui para a forma:

$$
Y_{2 \Delta t}=G^{2} \cdot Y_{0}+G \cdot I_{i 1}+I_{i 2}
$$

Generalizando a expressão (1), ela assume a forma:

$$
Y_{n \cdot \Delta t}=G^{n} \cdot Y_{0}+\sum_{i=0}^{n-1} G^{i} \cdot I_{(n-i)}
$$

em que

$n=$ número de períodos de prognose; e $Y_{t+} \Delta_{t}, \mathrm{G}, Y_{i t}, I_{i t}=$ já definidos anteriormente.

Para obtenção dos volumes a partir do número de árvores prognosticado para a vegetação, adotou-se o seguinte critério: os resultados obtidos em número de árvores foram transformados em área basal através da fórmula $\left[\left(\pi . D^{2}\right) / 40000 \times\right.$ número de árvores de cada classe diamétrica], em que $\mathrm{D}=$ valor central da classe diamétrica. A partir daí utilizou-se a equação desenvolvida por Mello (1999) para a região do estudo, com as seguintes características: $V=125,509270$. $\left(1-\mathrm{e}^{-0,051082 . \mathrm{G}}\right)^{1,423304}$. O coeficiente de determinação $\left(\mathrm{R}^{2}\right)$ e o erro-padrão residual $\left(S_{y x}\right)$ foram, respectivamente, $86,6 \%$ e $\pm 2,89 \mathrm{~m}^{3} / \mathrm{ha}$. Os dados resultantes da prognose em volume estão apresentados no Quadro 1.

\subsection{Estrutura de Custos e Preço da Madeira (lenha)}

Os custos de produção de lenha de vegetação do cerrado considerados neste estudo estão no Quadro 2. Não foi considerado, no caso da exploração do cerrado, o custo de implantação (após o corte da vegetação 
considera-se que haverá regeneração natural), o custo de combate à formiga, o custo de capina ou de qualquer outro trato cultural.

Os custos de produção de madeira de eucalipto para energia (lenha) usados neste estudo estão no Quadro 3. Considerou-se um ciclo de três cortes (7, 14 e 21 anos), e a produção volumétrica da primeira e da segunda talhadias correspondente a 90 e $80 \%$ da produção do alto fuste, respectivamente.

Como custo da terra considerou-se o custo de oportunidade de uso do fator representado pela taxa real de juros adotada na análise econômica. Foram considerados os preços de venda da lenha iguais a US\$ 5.70/st e US\$ 8.13/st para a madeira de vegetação de cerrado e de eucalipto, respectivamente. Estes preços são para lenha colocada no caminhão, incluindo os gastos com impostos e taxas, inclusive a taxa florestal. Eles foram fornecidos pela Companhia Mineira de Metais (CMM), grupo Votorantim, que explora e comercializa madeira de vegetação nativa de cerrado e de eucalipto.

\subsection{Análise Econômica}

Com base nos dados de custos de produção de madeira do Quadro 2, nos volumes do Quadro 1 e usando o preço de venda da madeira igual a US\$ 5.70/st, realizou-se a análise econômica dos diferentes regimes de manejo da vegetação do cerrado, que consistiu em determinar qual o tratamento mais viável economicamente, e em que ciclo de corte. Foram utilizados dois critérios de análise econômica referentes ao valor presente líquido de uma série infinita de rotações (VPL), que se diferenciam pela inclusão ou não do custo da terra, conforme descrito na seqüência (Rezende \& Oliveira, 1999). Para todo o estudo utilizou-se a taxa de desconto de $10 \%$ a.a.

Quadro 1 - Volumes retirado (RET) e remanescente (REM) (st/ha) dos tratamentos no ano em que houve a intervenção (1986), nas épocas em que foram realizados os inventários (fevereiro de 1996 e junho de 1998) e nos anos para os quais foram feitas as prognoses (outubro de 2000, fevereiro de 2003, junho de 2005 e outubro de 2007)

Table 1 - Volumes removed (REM) and remaining (st/ha) from the treatments in the intervention year (1986), inventory years February 1996 and June 1998)

\begin{tabular}{|l|c|c|c|c|c|c|c|c|c|c|}
\hline \multirow{2}{*}{$\begin{array}{c}\text { \% REM* } \\
\text { (ano) }\end{array}$} & \multicolumn{2}{|c|}{$50 \%$} & \multicolumn{2}{c|}{$70 \%$} & \multicolumn{3}{c|}{$80 \%$} & \multicolumn{3}{c|}{$90 \%$} \\
\cline { 2 - 11 } & REM & RET & REM & RET & REM & RET & REM & RET & REM & RET \\
\hline 1986 & 19,86 & 19,86 & 13,78 & 32,16 & 8,25 & 32,98 & 4,66 & 41,96 & 0,00 & 30,58 \\
Fev./1996 & 31,22 & 31,22 & 20,98 & 48,94 & 10,27 & 41,08 & 5,85 & 52,65 & 0,00 & 53,96 \\
Jun./1998 & 35,78 & 35,78 & 22,55 & 52,62 & 12,57 & 50,29 & 7,12 & 64,12 & 0,00 & 66,20 \\
Out./2000 & 36,24 & 36,24 & 22,14 & 51,65 & 12,79 & 51,16 & 7,33 & 65,97 & 0,00 & 66,78 \\
Fev./2003 & 42,00 & 42,00 & 24,98 & 58,29 & 15,30 & 61,19 & 8,70 & 78,31 & 0,00 & 78,92 \\
Jun./2005 & 48,87 & 48,87 & 28,03 & 65,40 & 18,30 & 73,19 & 10,40 & 93,60 & 0,00 & 92,58 \\
Out./2007 & 54,77 & 54,77 & 30,93 & 72,18 & 20,99 & 83,98 & 11,51 & 103,63 & 0,00 & 104,90 \\
\hline
\end{tabular}

* Porcentagem de remoção em área basal.

Quadro 2 - Custos de produção de madeira da vegetação do cerrado para lenha, incluindo taxas e impostos

Table 2 - Costs of production of wood of the vegetation of the savannah for firewood, including rates and imposed

\begin{tabular}{|l|c|c|}
\hline \multicolumn{1}{|c|}{ Item de Custo } & Ano de Ocorrência & Valor \\
\hline - Elaboração do projeto e topografia (US\$/ha) & ano 0 & 3.12 \\
- Construção de estradas e aceiros (US\$/ha) & ano 0 & 4.55 \\
- Conservação de estradas e aceiros (US\$/ha) & Anos de corte & 0.45 \\
- Corte (US\$/st) & Anos de corte & 0.94 \\
- Extração (baldeio) (US\$/st) & Anos de corte & 0.75 \\
- Carregamento (US\$/st) & Anos de corte & 0.62 \\
- Impostos e taxas, inclusive taxa florestal (US\$/st) & Anos de corte & 2.13 \\
- Custo da terra (US\$/ha) $(50.00 \times$ i) & Anual & 5.00 \\
\hline
\end{tabular}

Fonte: Companhia Mineira de Metais (CMM), grupo Votarantim. 
Quadro 3 - Custos de produção de madeira de eucalipto para energia (lenha), incluindo taxas e impostos Table 3 - Production costs of eucalyptus wood for energy (firewood), including rates and taxes

\begin{tabular}{|c|c|c|}
\hline Item de custo & Ano de Ocorrência & Valor \\
\hline - Infra-estrutura (US\$/ha) & Ano 0 & 33.10 \\
\hline - Preparo do terreno (US\$/ha) & Ano 0 & 302.66 \\
\hline - Plantio e replantio (US\$/ha) & Ano 0 & 254.38 \\
\hline - Combate às formigas (US\$/ha) & Ano 0 & 23.44 \\
\hline - Capina (US\$/ha) & Ano 1 & 84.23 \\
\hline - Capina / Roçada (US\$/ha) & Ano 2 & 38.33 \\
\hline - Capina / Roçada (US\$/ha) & Ano 3 & 38.33 \\
\hline - Condução de brotação (US\$/ha) & $1^{\circ}$ ano após o corte & 129.51 \\
\hline - Condução de brotação (US\$/ha) & $2^{\circ}$ ano após o corte & 25.70 \\
\hline - Colheita (US\$/st) & Anos de corte & 1.88 \\
\hline - Carregamento (US\$/st) & Anos de corte & 0.62 \\
\hline - Impostos e taxas, inclusive taxa florestal(US\$/st) & Anos de corte & 2.09 \\
\hline - Reforma (US\$/ha) & Final do ciclo & 208.00 \\
\hline - Combate às formigas (US\$/ha) & Anual & 9.34 \\
\hline - Conservação de estradas e aceiros (US\$/ha) & Anual & 1.78 \\
\hline - Custo da terra (US\$/ha) $(50.00 \mathrm{x}$ i) & Anual & 5.00 \\
\hline
\end{tabular}

Fonte: Companhia Mineira de Metais (CMM), grupo Votorantim.

\section{a) Valor Presente Líquido Geral (VPG)}

O "VPG" considera o custo de oportunidade pelo uso da terra, ou seja, a terra pode ser mantida para o presente povoamento, convertida em outro povoamento ou vendida para outros usos. Este critério pode ser expresso da seguinte forma:

$$
V P G=\frac{\sum_{n=0}^{t}\left(R_{n}-C_{n}\right) \cdot(1+t)^{t-n}-\left|L \cdot(1+i)^{t}-L\right|}{\left((1+i)^{t}-1\right)}
$$

em que $L=$ valor da terra; $t=$ idade de rotação; $R_{n}=$ receitas no ano $n ; C_{n}=$ custos no ano $n$; e $i=$ taxa de juros comparativa ou taxa mínima de atratividade.

O VPG concede para a terra somente o seu preço de mercado presente e que algum excedente é tratado como a renda capturada pelo proprietário acima da qual ele desejaria para remunerar seu salário e capital.

\section{b) Valor Esperado da Terra (VET)}

A pressuposição peculiar do modelo de renda do solo ou valor esperado da terra é que não existe acesso aos mercados de terra, isto é, a terra é um fator de produção fixo e, conseqüentemente, todos os excedentes econômicos resultam para a terra (Bentley \& Teenguarden, 1965). Estabelecendo-se o valor presente líquido geral (VPG) igual a zero, o VET pode ser obtido como se segue:

$$
V E T=\frac{\sum_{n=0}^{t}\left(R_{n}-C_{n}\right) \cdot(1+i)^{t-n}}{\left((1+i)^{t}-1\right)}
$$

O VPL calculado, levando em conta um horizonte infinito, foi usado também para verificar a viabilidade econômica de plantar eucalipto em áreas de cerrado, visando a produção de madeira para energia. Comparouse esta opção de uso da terra com a opção de manejo da vegetação do cerrado.

Para os regimes de manejo da vegetação do cerrado realizou-se uma análise de sensibilidade do VPL às variações no custo da terra, nos índices de produtividade, nos custos de produção de madeira e no preço da madeira, para estabelecer a influência destes fatores na viabilidade econômica dos tratamentos.

R. Árvore, Viçosa-MG, v.26, n.6, p.715-726, 2002 
Para a terra consideraram-se três níveis de custo, ou seja, zero, US\$ 50.00 e US\$100.00 por hectare.

Foram simulados os efeitos de aumentos de até $21 \%$ nos preços da madeira e de até $30 \%$ na produtividade da vegetação dos diversos tratamentos e ciclos de corte, assim como os efeitos de redução de até $20 \%$ nos custos de produção de madeira em relação aos níveis de custos originais.

Para o eucalipto foram simulados diversos níveis de produtividade de madeira para lenha e três níveis de valor para a terra (zero, US\$50.00 e US\$100.00 por hectare).

\section{RESULTADOS E DISCUSSÃO}

\subsection{Viabilidade Econômica dos Regimes de Manejo da Vegetação do Cerrado}

O Quadro 4 mostra como exemplo o fluxo de caixa para o tratamento em que se retirou $90 \%$ da área basal total, para um ciclo de corte de 10 anos e preço da terra igual a US\$50.00 por hectare.
O valor presente líquido (VPL), considerando um horizonte de planejamento infinito, é calculado da seguinte maneira:

$$
\begin{aligned}
V P L & =239.17+\frac{300.10}{(1.1)^{10}-1}-3.12-4.55-39.44-31.47-26.02 \\
& -89.37-\frac{(0.45+49.49+39.49+32.64+112.14)}{(1.1)^{10}-1}-\frac{5.00}{0.10}
\end{aligned}
$$

$V P L=36.54$

Os resultados para os outros tratamentos encontramse no Quadro 5. Adotando-se as produtividades encontradas pelos inventários realizados em 1996 e 1998 (ciclos de corte de 10 e 12 anos, respectivamente) e pelas prognoses para os anos de 2000, 2003, 2005 e 2007 (ciclos de corte de 14, 17, 19 e 21 anos, respectivamente), todos os tratamentos foram viáveis economicamente (apresentam VPL positivo), independentemente do ciclo de corte adotado, com exceção do tratamento de $50 \%$, em todos os ciclos, e o de $70 \%$ para os ciclos de 19 e 21 anos. Por exemplo, no tratamento em que houve retirada de $50 \%$ da área basal o VPL varia de US\$ -8.25, quando o ciclo

Quadro 4 - Fluxo de caixa para o tratamento em que se retirou 90\% da área basal total, considerando um ciclo de corte de 10 anos e preço da terra igual US\$ 50.00 por hectare

Table 4 -Cash flow for the management regime in which 50\% of the basal area was removed, considering a cutting cycle of

\begin{tabular}{|c|c|c|}
\hline Ano de Ocorrência & Discriminação & Valor (US\$/ha) \\
\hline \multicolumn{3}{|c|}{ Receitas } \\
\hline $0(1986)$ & - Venda de madeira & 239.17 \\
\hline $10(1996), 20(2006), \ldots \infty$ & - Venda da madeira & 300.10 \\
\hline \multicolumn{3}{|c|}{ Custos } \\
\hline $0(1986)$ & - Elaboração do projeto e topografia & 3.12 \\
\hline $0(1986)$ & - Construção de estradas e aceiros & 4.55 \\
\hline $0(1986)$ & - Corte & 39.44 \\
\hline $0(1986)$ & - Extração & 31.47 \\
\hline $0(1986)$ & - Carregamento & 26.02 \\
\hline $0(1986)$ & - Impostos e taxas & 89.37 \\
\hline $10(1996), 20(2006), \ldots \infty$ & - Conservação de estradas e aceiros & 0.45 \\
\hline $10(1996), 20(2006), \ldots \infty$ & - Corte & 49.49 \\
\hline $10(1996), 20(2006), \ldots \infty$ & - Extração & 39.49 \\
\hline $10(1996), 20(2006), \ldots \infty$ & - Carregamento & 32.64 \\
\hline $10(1996), 20(2006), \ldots \infty$ & - Impostos e taxas & 112.14 \\
\hline $1(1987), 2(1988), \ldots \infty$ & - Terra & 5.00 \\
\hline
\end{tabular}
10 years and land price of US\$50.00 per ha

* O símbolo " $\infty$ " indica que a receita ou o custo em questão repete infinitas vezes, em cada intervalo de tempo especificado. 
de corte é de 10 anos, a US\$-21.93, quando o ciclo de corte é de 21 anos.

Quadro 5 - Valor presente líquido por tratamento, para diversos ciclos de corte

Table 5 - Net present value for treatment for several cutting cycles

\begin{tabular}{|c|r|r|r|r|r|}
\hline \multirow{2}{*}{$\begin{array}{c}\text { Ciclo de } \\
\text { Corte }\end{array}$} & \multicolumn{5}{|c|}{ VPL (US\$/ha) / Tratamento } \\
\cline { 2 - 6 } & $50 \%$ & $70 \%$ & $80 \%$ & $90 \%$ & $100 \%$ \\
\hline 10 & -8.25 & 21.26 & 16.08 & 36.54 & 23.24 \\
12 & -11.77 & 13.65 & 13.31 & 32.77 & 19.66 \\
14 & -16.48 & 5.95 & 6.77 & 24.75 & 10.78 \\
17 & -19.71 & 0.86 & 2.79 & 20.48 & 5.28 \\
19 & -20.70 & -1.13 & 1.82 & 19.42 & 3.57 \\
21 & -21.93 & -3.01 & 0.35 & 15.53 & 1.44 \\
\hline
\end{tabular}

Para todos os tratamentos houve diminuição no valor do VPL à medida que o ciclo de corte aumentou. No tratamento $90 \%$ o VPL passa de US\$36.54, se o ciclo de corte for de 10 anos, para US\$15.53 para um ciclo de 21 anos.

Vale ressaltar que os tratamentos que implicaram a retirada de 90 e $100 \%$ da área basal apresentaram VPLs maiores que aquele de $70 \%$ de remoção da área basal, o que é bastante natural. Do ponto de vista de sustentação dos níveis de intervenção estudados, Mello (1999) identificou que $100 \%$ de remoção da área basal não acarreta perda de diversidade da flora, uma vez que todas as espécies apresentam alta capacidade de rebrotar. No entanto, dentro do conceito de sustentabilidade deve-se levar em conta que o componente fauna é igualmente importante para a manutenção de ecossistemas e que níveis intensos de remoção da área basal podem comprometer a fauna, pela escassez de alimento e abrigo. Portanto, não se recomenda sua aplicação em nenhuma circustância. Por último, estes níveis de intervenção conflitam com a legislação em vigor atualmente no Estado de Minas Gerais.

Pode-se, então, inferir que o ciclo econômico ótimo é de 10 anos. Não foram analisados ciclos mais curtos, por não ser permitido pela Lei Florestal do Estado de Minas Gerais.

Do ponto de vista de aplicação dos resultados obtidos neste estudo, um agricultor ou um empreendedor florestal que tem vegetação do cerrado com volume em torno de $46 \mathrm{~m}^{3} / \mathrm{ha}(13.78+32.16)$ (Quadro 1) pode, ao adotar uma intervenção de $70 \%$ da área basal, auferir um lucro de US\$21.26/ha para o ciclo de corte de
10 anos, se custos e preços idênticos ao deste estudo forem considerados em sua intervenção. Este mesmo raciocínio pode ser adotado para áreas que tiverem características similares à volumetria existente em 1986, para os outros tratamentos.

O preço da terra afeta bastante o VPL dos tratamentos, conforme mostra o Quadro 6. No tratamento em que foi realizado corte raso, considerando um ciclo de corte de 10 anos, o VPL passa de US\$ 73.24, se o preço da terra for igual a zero, para US\$ -26.76 , se o preço for de US\$100.00/ha, ou seja, o prejuízo aumenta cerca de $373,69 \%$.

Para o preço da terra igual a zero todos os tratamentos foram viáveis economicamente, para todos os ciclos de corte. Já para o preço da terra igual a US\$100.00/ha todos os tratamentos apresentaram-se inviáveis economicamente.

De acordo com Silva et al. (1997), o custo de oportunidade da terra é o retorno que pode ser obtido caso os investimentos em terra fossem aplicados em outra alternativa. Para este estudo, o custo de oportunidade de uso da área que pertence a uma empresa de reflorestamento é o valor que poderia ser obtido caso a área fosse utilizada para outros fins, como para plantio de eucalipto ou aplicação financeira.

Os investimentos em terra para fins florestais são altos e devem ser considerados na avaliação econômica.

O Quadro 7 mostra as simulações de aumentos porcentuais na produtividade da vegetação do cerrado para todos os tratamentos e ciclos de corte, em relação às produtividades encontradas nos inventários aos $10 \mathrm{e}$ 12 anos (1996 e 1998) e pelas prognoses para 14, 17, 19 e 21 anos (2000, 2003, 2005 e 2007). Como era esperado, aumentando-se a produtividade dos tratamentos o VPL aumenta. O tratamento 50\% passa a ter VPL positivo no ciclo de corte de 10 anos quando há aumento de $20 \%$ ou mais na produtividade, enquanto no ciclo de 12 anos é necessário o aumento de $30 \%$ na produtividade para que o VPL fique positivo. Em todas as outras situações o VPL deste tratamento é negativo.

Segundo Rezende et al. (1986), investimentos no manejo do cerrado, visando aumentar a produtividade, poderiam ser mais compensadores do que investir no plantio de eucalipto. Contudo, deve-se considerar que na época em que este estudo foi realizado a situação era diferente da atual, pois o custo de implantação de povoamentos de eucalipto caiu de mais de US\$1,000.00/ha 
Quadro 9 - Valor presente líquido por tratamento e ciclo de corte, para diferentes preços da madeira Table 9 - Net present value for treatment and cutting cycle for different wood prices

\begin{tabular}{|c|c|c|c|c|c|c|c|}
\hline \multirow{3}{*}{$\begin{array}{c}\text { Tratamento } \\
(\%)\end{array}$} & \multirow{3}{*}{$\begin{array}{l}\text { Preço da } \\
\text { Madeira } \\
\text { (US\$/st) }\end{array}$} & \multicolumn{6}{|c|}{ VPL (US\$/ha) } \\
\hline & & \multicolumn{6}{|c|}{ Ciclo de Corte (anos) } \\
\hline & & 10 & 12 & 14 & 17 & 19 & 21 \\
\hline \multirow{3}{*}{50} & 5.70 & -8.25 & -11.27 & -16.48 & -19.71 & -20.70 & -21.93 \\
\hline & 6.30 & 15.42 & 10.18 & 3.20 & -1.57 & -3.05 & -4.88 \\
\hline & 6.90 & 39.09 & 32.14 & 22.89 & 16.56 & 14.60 & 12.17 \\
\hline \multirow{3}{*}{70} & 5.70 & 21.26 & 13.65 & 5.95 & 0.86 & -1.13 & -3.01 \\
\hline & 6.30 & 58.98 & 47.41 & 36.33 & 28.78 & 25.84 & 23.05 \\
\hline & 6.90 & 96.70 & 81.77 & 66.70 & 56.70 & 52.80 & 49.12 \\
\hline \multirow{3}{*}{80} & 5.70 & 16.08 & 13.31 & 6.77 & 2.79 & 1.82 & 0.35 \\
\hline & 6.30 & 51.33 & 47.20 & 37.53 & 31.63 & 30.19 & 28.01 \\
\hline & 6.90 & 86.59 & 81.10 & 68.29 & 60.48 & 58.57 & 55.67 \\
\hline \multirow{3}{*}{90} & 5.70 & 36.54 & 32.77 & 24.75 & 19.42 & 20.41 & 15.53 \\
\hline & 6.30 & 81.54 & 75.94 & 64.08 & 56.19 & 56.56 & 50.42 \\
\hline & 6.90 & 126.54 & 119.10 & 103.40 & 92.95 & 92.71 & 85.31 \\
\hline \multirow{3}{*}{100} & 5.70 & 23.24 & 19.66 & 10.78 & 5.28 & 3.57 & 1.44 \\
\hline & 6.30 & 61.90 & 56.58 & 43.45 & 35.30 & 32.78 & 29.62 \\
\hline & 6.90 & 100.56 & 93.50 & 76.12 & 65.33 & 61.99 & 57.81 \\
\hline
\end{tabular}

Quadro 7 - Valor presente líquido por tratamento e ciclo de corte, para vários níveis de aumento na produtividade de madeira para energia (lenha)

Table 7 - Net present value for treatment and cutting cycle for several increase levels in wood productivity for energy (firewood)

\begin{tabular}{|c|c|c|c|c|c|c|c|}
\hline \multirow{3}{*}{$\begin{array}{c}\text { Tratamento } \\
(\%)\end{array}$} & \multirow{3}{*}{$\begin{array}{c}\text { Aumento na } \\
\text { Produtividade } \\
(\%)\end{array}$} & \multicolumn{6}{|c|}{ VPL (US\$/ha) } \\
\hline & & \multicolumn{6}{|c|}{ Ciclo de Corte (anos) } \\
\hline & & 10 & 12 & 14 & 17 & 19 & 21 \\
\hline \multirow{4}{*}{50} & 0 & -8.25 & -11.77 & -16.48 & -19.71 & -20.70 & -21.93 \\
\hline & 10 & -3.28 & -7.16 & -12.35 & -15.90 & -16.99 & -18.35 \\
\hline & 20 & 1.69 & -2.55 & -8.22 & -12.09 & -13.29 & -14.77 \\
\hline & 30 & 6.67 & 2.06 & -4.08 & -8.28 & -9.58 & -11.19 \\
\hline \multirow{4}{*}{70} & 0 & 21.26 & 13.65 & 5.95 & 0.86 & -1.13 & -3.01 \\
\hline & 10 & 29.18 & 20.80 & 12.33 & 6.72 & 4.53 & 2.46 \\
\hline & 20 & 37.10 & 27.95 & 18.71 & 12.58 & 10.20 & 7.94 \\
\hline & 30 & 45.02 & 35.10 & 25.09 & 18.45 & 15.86 & 13.41 \\
\hline \multirow{4}{*}{80} & 0 & 16.08 & 13.31 & 6.77 & 2.79 & 1.82 & 0.35 \\
\hline & 10 & 23.48 & 20.42 & 13.23 & 8.85 & 7.78 & 6.16 \\
\hline & 20 & 30.89 & 27.54 & 19.69 & 14.90 & 13.74 & 11.96 \\
\hline & 30 & 38.29 & 34.66 & 26.15 & 20.96 & 19.70 & 17.77 \\
\hline \multirow{4}{*}{90} & 0 & 36.54 & 32.77 & 24.75 & 19.42 & 20.41 & 15.53 \\
\hline & 10 & 45.99 & 41.83 & 33.01 & 27.15 & 28.22 & 22.86 \\
\hline & 20 & 55.44 & 50.90 & 41.27 & 34.87 & 36.04 & 30.18 \\
\hline & 30 & 64.89 & 59.96 & 49.53 & 42.59 & 43.86 & 37.51 \\
\hline \multirow{4}{*}{100} & 0 & 23.24 & 19.66 & 10.78 & 5.28 & 3.57 & 1.44 \\
\hline & 10 & 31.36 & 27.41 & 17.64 & 11.58 & 9.71 & 7.36 \\
\hline & 20 & 39.48 & 35.16 & 24.50 & 17.89 & 15.84 & 13.28 \\
\hline & 30 & 47.60 & 42.92 & 31.36 & 24.19 & 21.97 & 19.20 \\
\hline
\end{tabular}


para menos de US\$600.00/ha, atualmente. A produtividade destes povoamentos subiu de mais ou menos 20 st/ha.ano para 35-40 st/ha.ano e o custo de produção de carvão de eucalipto, em consequência, caiu de US\$ $21.00 / \mathrm{m}^{3}$ para mais ou menos US\$ $11.00 / \mathrm{m}^{3}$.

Reduzindo os custos de produção de madeira em até $10 \%$ com relação aos custos originais (Quadro 8), é possível fazer com que todos os tratamentos sejam lucrativos, com exceção do tratamento $50 \%$, para os ciclos de corte de 17,19 e 21 anos. Por exemplo, neste tratamento a queda de $10 \%$ nos custos faz com que, no ciclo de corte de 10 anos, o VPL passe de US\$ -8.25 para US\$ 15.06.

O Quadro 9 mostra que aumentos no preço da madeira proporcionam aumentos no VPL dos tratamentos, em todos os ciclos de corte. No tratamento de $90 \%$ e ciclo de corte de 10 anos, se o preço da madeira aumentar de US\$ 5.70 para US\$ 6.30/st o VPL passa de US\$ 36.54 para US\$ 81.54. Já no tratamento 50\% o VPL torna-se positivo, em todos os ciclos de corte, quando o preço da madeira aumenta para US\$ 6.90/st.

\subsection{Viabilidade Econômica do Plantio de Eucalipto em Regiões de Cerrado}

O Quadro 10 mostra como exemplo o fluxo de caixa para o reflorestamento com eucalipto, considerando o incremento médio de $30 \mathrm{st} / \mathrm{ha} /$ ano para o alto fuste (corte aos 7 anos) e a produção volumétrica da primeira (corte aos 14 anos) e segunda (corte aos 21 anos) talhadias, correspondente a 90 e $80 \%$ da produção do alto fuste, respectivamente. O valor presente líquido (VPL), considerando o horizonte de planejamento infinito, é calculado da seguinte maneira:

$$
\begin{aligned}
& V P L=\frac{\frac{1,707.30(1.1)^{21}}{(1.1)^{21}-1}}{(1.1)^{7}}+\frac{\frac{1,536.57(1.1)^{21}}{(1.1)^{21}-1}}{(1.1)^{14}}+\frac{1,365.48}{(1.1)^{21}-1}-33.10-302.66-254.38 \\
& -\frac{23.44(1.1)^{21}}{(1.1)^{21}-1}-\frac{\frac{84.23(1.1)^{21}}{(1.1)^{21}-1}}{(1.1)}-\frac{\frac{38.33(1.1)^{21}}{(1.1)^{21}-1}}{(1.1)^{2}}-\frac{\frac{38.33(1.1)^{21}}{(1.1)^{21}-1}}{(1.1)^{3}}-\frac{\frac{963.90(1.1)^{21}}{(1.1)^{21}-1}}{(1.1)^{7}} \\
& -\frac{\frac{867.51(1.1)^{21}}{(1.1)^{21}-1}}{(1.1)^{14}}-\frac{771.12}{(1.1)^{21}-1}-\frac{129.31(1.1)^{13}+129.51(1.1)^{6}}{(1.1)^{21}-1}-\frac{208.00}{(1.1)^{21}-1} \\
& -\frac{25.70(1.1)^{12}+25.70(1.1)^{5}}{(1.1)^{21}-1}-\frac{9.34}{0.10}-\frac{1.78}{0.10}-\frac{5.00}{0.10}
\end{aligned}
$$

$V P L=-356.79$

O Quadro 11 mostra outros valores de VPL simulando mudanças no incremento médio anual (IMA) e preço da terra. Com base nos custos e nos preços da madeira adotados neste estudo e considerando o preço da terra igual a US\$ 50.00, o plantio de eucalipto em áreas de cerrado só é uma opção viável economicamente se a produtividade da floresta for de pelo menos 45st/ ha.ano (VPL igual a US\$12.06), o que demonstra a

\begin{tabular}{|c|c|c|c|c|c|c|c|}
\hline \multirow{3}{*}{$\begin{array}{c}\text { Tratamento } \\
(\%)\end{array}$} & \multirow{3}{*}{$\begin{array}{c}\text { Redução dos } \\
\text { Custos (\%) }\end{array}$} & \multicolumn{6}{|c|}{ VPL (US\$/ha) } \\
\hline & & \multicolumn{6}{|c|}{ Ciclo de Corte (anos) } \\
\hline & & 10 & 12 & 14 & 17 & 19 & 21 \\
\hline \multirow{3}{*}{50} & 0 & -8.25 & -11.27 & -16.48 & -19.71 & -20.70 & -21.93 \\
\hline & 10 & 15.06 & 10.26 & 3.87 & -0.51 & -1.86 & -3.54 \\
\hline & 20 & 38.37 & 32.30 & 24.22 & 18.69 & 16.97 & 14.85 \\
\hline \multirow{3}{*}{70} & 0 & 21.26 & 13.65 & 5.95 & 0.86 & -1.13 & -3.01 \\
\hline & 10 & 54.97 & 44.64 & 34.21 & 27.30 & 24.60 & 22.05 \\
\hline & 20 & 88.68 & 75.63 & 62.47 & 53.74 & 50.33 & 47.11 \\
\hline \multirow{3}{*}{80} & 0 & 16.08 & 13.31 & 6.77 & 2.79 & 1.82 & 0.35 \\
\hline & 10 & 47.96 & 44.18 & 35.31 & 29.91 & 28.59 & 26.59 \\
\hline & 20 & 79.85 & 75.05 & 63.86 & 57.03 & 55.36 & 52.83 \\
\hline \multirow{3}{*}{90} & 0 & 36.54 & 32.77 & 24.75 & 19.42 & 20.41 & 15.53 \\
\hline & 10 & 75.64 & 70.50 & 59.64 & 52.41 & 52.71 & 47.12 \\
\hline & 20 & 114.73 & 108.23 & 94.52 & 85.39 & 85.02 & 78.72 \\
\hline \multirow{3}{*}{100} & 0 & 23.24 & 19.66 & 10.78 & 5.28 & 3.57 & 1.44 \\
\hline & 10 & 57.64 & 52.77 & 40.74 & 33.27 & 30.96 & 28.07 \\
\hline & 20 & 92.05 & 85.88 & 70.70 & 61.27 & 58.35 & 54.70 \\
\hline
\end{tabular}
importância de melhorar a produtividade dos plantios.

Quadro 8 - Valor presente líquido por tratamento e ciclo de corte, para vários níveis de redução nos custos de produção Table 8 - Net present value for treatment and cutting cycle for several production cost reduction levels 
Quadro 9 - Valor presente líquido por tratamento e ciclo de corte, para diferentes preços da madeira.

Table 9 - Net present value for treatment and cutting cycle for different wood prices

\begin{tabular}{|c|c|c|c|c|c|c|c|}
\hline \multirow{3}{*}{$\begin{array}{c}\text { Tratamento } \\
(\%)\end{array}$} & \multirow{3}{*}{$\begin{array}{l}\text { Preço da } \\
\text { Madeira } \\
\text { (US\$/st) }\end{array}$} & \multicolumn{6}{|c|}{ VPL (US\$/ha) } \\
\hline & & \multicolumn{6}{|c|}{ Ciclo de Corte (anos) } \\
\hline & & 10 & 12 & 14 & 17 & 19 & 21 \\
\hline \multirow{3}{*}{50} & 5.70 & -8.25 & -11.27 & -16.48 & -19.71 & -20.70 & -21.93 \\
\hline & 6.30 & 15.42 & 10.18 & 3.20 & -1.57 & -3.05 & -4.88 \\
\hline & 6.90 & 39.09 & 32.14 & 22.89 & 16.56 & 14.60 & 12.17 \\
\hline \multirow{3}{*}{70} & 5.70 & 21.26 & 13.65 & 5.95 & 0.86 & -1.13 & -3.01 \\
\hline & 6.30 & 58.98 & 47.41 & 36.33 & 28.78 & 25.84 & 23.05 \\
\hline & 6.90 & 96.70 & 81.77 & 66.70 & 56.70 & 52.80 & 49.12 \\
\hline \multirow{3}{*}{80} & 5.70 & 16.08 & 13.31 & 6.77 & 2.79 & 1.82 & 0.35 \\
\hline & 6.30 & 51.33 & 47.20 & 37.53 & 31.63 & 30.19 & 28.01 \\
\hline & 6.90 & 86.59 & 81.10 & 68.29 & 60.48 & 58.57 & 55.67 \\
\hline \multirow{3}{*}{90} & 5.70 & 36.54 & 32.77 & 24.75 & 19.42 & 20.41 & 15.53 \\
\hline & 6.30 & 81.54 & 75.94 & 64.08 & 56.19 & 56.56 & 50.42 \\
\hline & 6.90 & 126.54 & 119.10 & 103.40 & 92.95 & 92.71 & 85.31 \\
\hline \multirow{3}{*}{100} & 5.70 & 23.24 & 19.66 & 10.78 & 5.28 & 3.57 & 1.44 \\
\hline & 6.30 & 61.90 & 56.58 & 43.45 & 35.30 & 32.78 & 29.62 \\
\hline & 6.90 & 100.56 & 93.50 & 76.12 & 65.33 & 61.99 & 57.81 \\
\hline
\end{tabular}

Quadro 10 - Fluxo de caixa para o reflorestamento com eucalipto, considerando um ciclo de três cortes (7, 14 e 21 anos) Table 10 - Cash flow for eucalyptus plantation, based on a seven year-rotation and three coppicings (7, 14 and 21 years)

\begin{tabular}{|l|l|r|}
\hline \multicolumn{1}{|c|}{ Ano de Ocorrência } & \multicolumn{1}{|c|}{ Discriminação } & \multicolumn{1}{c|}{$\begin{array}{l}\text { Valor } \\
\text { (US\$/ha) }\end{array}$} \\
\hline \multicolumn{2}{|c|}{ Receitas } & $1,707.30$ \\
\hline $7,28,49, \ldots \infty$ & - Venda da madeira do alto fuste & $1,536.57$ \\
$14,35,56, \ldots \infty$ & - Venda da madeira da primeira talhadia & $1,365.84$ \\
$21,42,63, \ldots \infty$ & - Venda da madeira da segunda talhadia & 33.10 \\
\hline \multicolumn{2}{|c|}{} & 302.66 \\
\hline 0 & - Infra-estrutura & 254.38 \\
0 & - Preparo do terreno & 23.44 \\
0 & - Plantio e replantio & 84.23 \\
$0,21,42, \ldots \infty$ & - Combate a formigas & 38.33 \\
$1,22,43, \ldots \infty$ & - Capina & 38.33 \\
$2,23,44, \ldots \infty$ & - Capina/roçada & 963.90 \\
$3,24,45, \ldots \infty$ & - Capina/roçada & 867.51 \\
$7,28,49, \ldots \infty$ & - Colheita, carregamento. impostos e taxas referentes à madeira do alto fuste & 771.12 \\
$14,35,56, \ldots \infty$ & - Colheita, carregamento. impostos e taxas referentes à madeira da primeira talhadia & 129.51 \\
$21,42,63, \ldots \infty$ & - Colheita, carregamento. impostos e taxas referentes à madeira da segunda talhadia & 25.70 \\
8 e $15 ; 29$ e $36 ; \ldots \infty$ & - Condução da brotação & 208.00 \\
9 e $16 ; 30$ e $37 ; \ldots \infty$ & - Condução da brotação & 9.34 \\
$21,42,63 \ldots \infty$ & - Reforma & 1.78 \\
$1,2,3, \ldots \infty$ & - Combate às formigas & 5.00 \\
$1,2,3, \ldots \infty$ & - Conservação de estradas e aceiros & \\
$1,2,3, \ldots \infty$ & - Terra & \\
\hline
\end{tabular}

* O símbolo " $\infty$ " indica que a receita ou o custo em questão repete infinitas vezes, em cada intervalo de tempo especificado.

** Considerou-se incremento médio anual de $30 \mathrm{st} / \mathrm{ha} / \mathrm{ano}$ para o alto fuste e a produção volumétrica da primeira e da segunda talhadias, correspondente a 90 e $80 \%$ da produção do alto fuste, respectivamente. 
Quadro 11 - Valor presente líquido para o plantio de eucalipto em área de cerrado, considerando diversos níveis de produtividade e de preços da terra

Table 11 - Net present value for eucalyptus plantation in savannah area for several land productivity and price levels

\begin{tabular}{|c|r|r|r|}
\hline \multirow{2}{*}{$\begin{array}{c}\text { Incremento Médio Anual } \\
\text { (IMA) (st/ha/ano)* }\end{array}$} & \multicolumn{3}{|c|}{ VPL/Preço da terra (US\$/ha) } \\
\cline { 2 - 4 } & \multicolumn{1}{|c|}{0.00} & 50.00 & 100.00 \\
\hline 30 & -306.79 & -356.79 & -406.79 \\
35 & -183.84 & -233.84 & -283.84 \\
40 & -60.89 & -110.89 & -160.89 \\
45 & 62.06 & 12.06 & -37.94 \\
50 & 185.02 & 135.02 & 85.02 \\
55 & 307.97 & 257.97 & 207.97 \\
\hline
\end{tabular}

* Refere-se ao IMA do $1^{\circ}$ corte (alto fuste). Para o IMA da $1^{\underline{a}}$ e $2^{a}$ talhadia foram consideradas quedas de 10 e $20 \%$ em relação ao IMA do alto fuste, respectivamente.

Segundo Novais et al. (1996), associando-se fertilização do solo e utilização de espécies/genótipos adequados, são facilmente obtidos de 25 a $30 \mathrm{~m}^{3} / \mathrm{ha}$.ano de madeira de eucalipto em solos de cerrado.

Berger (1983) estudou a rentabilidade dos reflorestamentos em função da produtividade e do preço da terra, concluindo que acréscimos de $40 \%$ na produtividade podem originar aumentos de até $150 \%$ na renda do empreendimento e que, para um dado preço da madeira e taxa de desconto, o aumento na renda do empreendimento é mais que proporcional ao acréscimo da produtividade.

Comparando os dados do Quadro 11, referentes ao preço da terra igual a US\$50.00, com os dados do Quadro 5 pode-se inferir que do ponto de vista econômico o investimento em plantio de eucalipto só é mais interessante que o manejo da vegetação do cerrado para níveis altos de produtividade do eucalipto. Por exemplo, para produtividade de 45st/ha.ano, o VPL do plantio de eucalipto é igual a US\$12.06, mas no ciclo de corte de 10 anos todos os tratamentos apresentam VPL maior que este, exceto o tratamento 50\%, cujo VPL é negativo (US\$-8.25). Mas se a produtividade subir para 50st/ha.ano o VPL passa a US\$ 135.02, tornando a atividade mais viável economicamente que todos os tratamentos.

\section{CONCLUSÕES}

Nas condições específicas em que se desenvolveu este estudo, pode-se concluir:
- A exploração da vegetação do cerrado, visando produzir madeira para lenha, é viável economicamente para os níveis de intervenção 70, 80, 90 e $100 \%$ de retirada de área basal.

- Dos ciclos de corte estudados, o melhor é o de 10 anos.

- Pequenos aumentos no preço da madeira da vegetação do cerrado causam grandes alterações na lucratividade dos regimes de manejo.

- O custo da terra é significativo na produção de lenha da vegetação do cerrado, o que evidencia que os planos de manejo desta vegetação podem ser mais viáveis economicamente se forem implementados em regiões onde o preço da terra é baixo.

- O plantio de eucalipto em região de cerrado só é mais interessante que manejar a vegetação se a produtividade do eucalipto for maior do que $45 \mathrm{st} / \mathrm{ha}$.ano.

\section{REFERÊNCIAS BIBLIOGRÁFICAS}

ABRACAVE - Informativo da Associação Brasileira de Florestas Renováveis. v. 7, n. 23, jan - mar/98.

BENTLEY, W.; TEENGUARDEN, D. Financial maturity: a theory review. Forest Science, v. 11, n. 3, p. 76-87, 1965.

BERGER, R. Preço máximo de terras para reflorestamento sua importância na viabilidade de empreendimentos florestais. IPEF, n. 23, p. 11-30, 1983.

BUONGIORNO, J.; MICHIE, B. R. A matrix model of uneven-aged forest management. Forest Science, v. 26, n. 4 , p. $609-625,1980$.

CABRAL, C. E. C. Desenvolvimento de um sistema computacional para simular e comparar economicamente alternativas de manejo de plantações florestais. ViçosaMG: UFV, 1990. 108 p. Dissertação (Mestrado em Ciências Florestais) - Universidade Federal de Viçosa, 1990.

LEITE, A. P. Avaliação silvicultural e econômica da vegetação do cerrado e eucalipto submetida a diferentes regimes de manejo na região noroeste de MG. Lavras: UFLA, 1998. 99 p. Dissertação (Mestrado em Engenharia Florestal) - Universidade Federal de Lavras, 1998.

R. Árvore, Viçosa-M G, v.26, n.6, p.715-726, 2002 
MELLO, A. A. Estudo silvicultural e da viabilidade econômica do manejo da vegetação do cerrado. Lavras: UFLA, 1999. 187 p. Dissertação (Mestrado em Engenharia Florestal) - Universidade de Lavras, 1999.

NOVAIS, R. F.; BARROS, N. F.; COSTA, L. M. Aspectos nutricionais e ambientais do eucalipto. Silvicultura, n. 68, p. 10-17, 1996.

PULZ, F. A. et al. Acuracidade da predição da distribuição diamétrica de uma floresta inequiânea com a matriz de transição. Cerne, v. 3, n. 1, p. 77-96, 1999.
REZENDE, J. L. P.; OLIVEIRA, A. D. Avaliação econômica de projetos florestais. Lavras: UFLA/FAEPE, 120 p. 1999.

REZENDE, J. L. P.; VALE, A. B.; MINETTE, L. Estudo comparativo da produção de carvão da madeira da vegetação nativa e de Eucalyptus spp. Viçosa: SIF/IBDF 1986. 45 p. (Relatório)

SILVA, M. L.; LIMA JÚNIOR, V. B.; REZENDE, J. L. P. Custo da terra. Viçosa-MG: UFV, 1997. 19 p. (não publicado). 